\title{
Klopfen gegen die Angst
}

\section{Die MET-KLOPFTHERAPIE als Kurzzeitmethode gegen belastende Gefühle und Glaubenssätze.}

Rainer und Regina Franke

\section{KURZ GEFASST}

1 Die MET-Klopftherapie wurde vom Autor als Synthese chinesischer Akupressurtechniken und westlicher Psychotherapie als schnell wirksame Kurzzeitbehandlung entwickelt.

2 Durch Beklopfen sechs charakteristischer Punkte und gleichzeitigem Fokussieren des belastenden Gefühls löst sich dieses zunehmend auf.

3 Die Methode eignet sich unter anderem bei Ängsten, Trauer, Zwängen und Selbstwertstörungen und lässt sich sehr gut mit anderen harmonisierenden Techniken wie Atemübungen oder Punktmassage kombinieren.

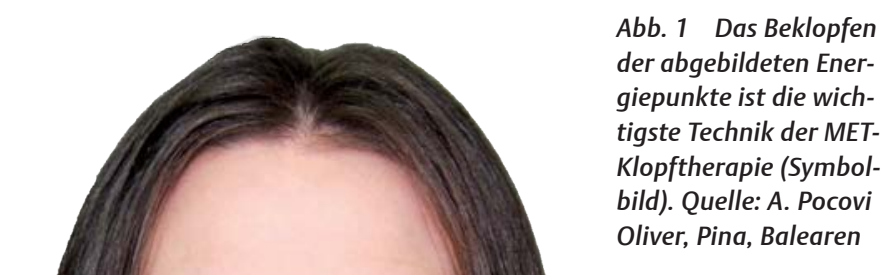

Glaubenssätze und Ängste einfach manuell auflösen? Als ich 2001 das Beklopfen von Meridianpunkten als psychotherapeutisches Instrument kennenlernte, blieb ich skeptisch: Als klassischer Diplom-Psychologe und Gestaltpsychotherapeut war ich es gewohnt, an Problemstellungen mit den bekannten Methoden über einen längeren Zeitraum zu arbeiten. Für mich stand fest: Veränderungen erfordern Zeit und die konsequente Mitwirkung der Klienten. Jedoch schon in der ersten Fortbildung zur EnergieFeld-Therapie machte ich eine Erfahrung, die mich nachhaltig beeindruckte: Eine bei mir seit 40 Jahren bestehende, therapieresistente Angst vor uniformierten Polizisten löste sich in Minuten auf. Auch kleinere Versuche mit der Familie und an 30 Freiwilligen zeigten deutliche Wirkung. Ob Spinnenphobie, Höhen- oder Versagensangst: Ängste lösten sich unter der Behandlung in vielen Fällen auf und wurden von Ruhe, Gelassenheit und Zuversicht abgelöst. Daraufhin begann ich, die Klopftherapie als alte Methode der Traditionellen Chinesischen Medizin (TCM) mit meiner psychotherapeutischen Arbeit zu verknüpfen. Im Jahr 2003 war die Meridian-Energie-Technik (MET) geboren.

\section{Das Prinzip: Durch Beklopfen Blockaden lösen}

Das Prinzip der Therapie: Durch sanftes Beklopfen bestimmter Akupunkturpunkte lösen sich energetische Blockaden. Nach der TCM gilt ein Mensch als gesund an Leib und Seele, wenn die Energie in den Meridianen frei fließen kann. Behinderungen und Blockaden im Energiefluss führen umgekehrt zu körperlichen und seelischen Erkrankungen. Schon vor ca. 5000 Jahren wurden deshalb bestimmte Meridianpunkte mit den Fingerspitzen beklopft, um den Energiefluss wiederherzustellen.

Die MET-Klopftherapie ist für verschiedene in einer psychotherapeutischen Praxis auftretende Probleme anwendbar. In die Methode fließen verschiedene Elemente und Techniken unterschiedlicher therapeutischer Schulen ein, darunter Gestalttherapie nach Fritz und Laura Perls (Fühlen im Hier und Jetzt), die Arbeit von Alice Miller (frühkindliche Traumatisierungen berücksichtigen), der systemische Ansatz (Einbeziehen des Beziehungssystems), sowie Sichtweisen und Techniken der energetischen Psychologie (Thought Field Therapy nach Callahan). 


\section{Angst, Schmerz und Glaubenssätze in ihr Gegenstück überführen}

MET verknüpft profundes therapeutisches Wissen mit dem energetischen Ansatz der TCM. Die Methode eignet sich insbesondere bei belastenden Emotionen wie Ängsten, Trauer, Kummer, Scham, Schuldgefühlen, Eifersucht, Wut oder Zwängen. Auch mangelndes Selbstwertgefühl, Ermüdung, Burnout-Erscheinungen oder Resignation zählen zu den Indikationen. Dies gilt auch für blockierende Glaubenssätze wie „ich bin wertlos“, „Geld stinkt“ oder „wahre Liebe gibt es nicht". Sind sie erst einmal bewusst geworden, können auch sie „beklopft“ und in ihr positives, stärkendes Gegenteil verwandelt werden.

Körperliche Symptome wie Asthma, Allergien, Kopf-, Rückenund sonstige Schmerzen sowie psychosomatische Folgen von Traumata lassen sich mit MET ebenfalls günstig beeinflussen. Dies gilt auch für suchtmäßiges Verlangen, zum Beispiel nach Kaffee, Süßigkeiten (Schokolade), Alkohol oder Nikotin.

Im Coaching kann MET zur Leistungssteigerung in Sport und Beruf eingesetzt werden. Denn auch hier beruhen Leistungsblockaden häufig auf Ängsten, Zweifeln oder blockierenden Überzeugungen und Glaubenssätzen, die sich durch das Beklopfen der Meridianpunkte nachhaltig verändern lassen.

In der therapeutischen Praxis findet MET auch Anwendung bei tieferen therapeutischen Problemen einschließlich Depressionen und Essstörungen, die sich mit klassischen Therapiemethoden nur schwer behandeln lassen.

\section{Wie genau funktioniert die MET-Klopftherapie?}

Bei MET werden belastende negative Gefühle durch das Beklopfen ausgewählter Akupunkturpunkte aufgelöst oder transformiert. Die Theorie dahinter: Jedes negative Gefühl wird durch eine Unterbrechung im Energie(-Meridian-)-System des Körpers hervorgerufen. Demnach führt nicht eine traumatische Erfahrung zu Befindlichkeitsstörungen, sondern eine energetische Unterbrechung im Energiesystem des Körpers im Moment der Traumatisierung. Anfänglich waren es 14 Akupunkturpunkte. Durch die reichhaltige Praxiserfahrung konnten wir feststellen, dass im Wesentlichen der 6. Akupunkturpunkt, der sich unterhalb der Schlüsselbeingelenke befindet, ausreicht, um diese emotionalen Belastungen aufzulösen. Eine einfache Form der Technik können Laien in einem mehrstündigen Kurs erlernen. Die therapeutische Anwendung benötigt eine profundere Ausbildung, die sich über mindestens zwei Jahre erstreckt. Diese beschließt dann je nach beruflicher Ausrichtung als MET-Therapeut ${ }^{\circledR}$ (zum Beispiel für Ärzte, Zahnärzte, Psychologen oder Heilpraktiker) oder MET-Coach ${ }^{\circledR}$ (für alle beratenden Berufe und Coaches).

\section{MET-Trauma-Technik: Gefühle dosiert abrufen und umschreiben}

Die MET-Trauma-Technik hat sich zur Behandlung von Traumafolgen bewährt. Bei Traumabehandlungen muss allerdings darauf geachtet werden, dass der Klient nicht zu sehr in das traumatische

\section{HINTERGRUNDWISSEN}

\section{Schutz vor überflutenden Gefühlen: Die Leinwand- und Containertechnik}

Bei der Leinwandtechnik projiziert der Klient das belastende Bild auf eine imaginäre Leinwand, die zu Beginn der Behandlung so weit weggeschoben wird, dass das Bild etwa briefmarkenklein ist. Somit verursacht es nur noch eine geringe emotionale Belastung. Im Lauf der Behandlung wird diese Leinwand immer näher herangezogen und die jeweiligen emotionalen Belastungen bei jedem Schritt aufgelöst. Erst wenn der Klient bereit ist, wird die Leinwand wieder näher herangezogen, bis sie sich nur noch in einem Meter Abstand vor ihm befindet.

Am Ende der Behandlung kann die Containertechnik angewandt werden, wenn das Trauma noch nicht komplett aufgelöst wurde. Dann kann der Klient das emotional noch belastende Bild in einen virtuellen Container packen und diesen verschließen. Den Schlüssel für das Schloss behält er mit der Bemerkung, dass er allein den Zeitpunkt für die nächste Behandlung bestimmt.

Erlebnis eintaucht und somit eine Retraumatisierung erfährt. Er darf die auftauchenden Gefühle maximal nur in der Intensität erleben, die er auch verkraften kann, und nicht von ihnen überschwemmt werden. Dies gewährleisten unter anderem die Leinwandtechnik sowie die Containertechnik (siehe Kasten).

Erstere stellt sicher, dass die belastenden Gefühle in kleinen Dosierungen hochkommen und für den Patienten aushaltbar gelöst werden können. Dank der Containertechnik kann eine METTraumabehandlung jederzeit problemlos unterbrochen und zu einem späteren Zeitpunkt fortgesetzt werden. Die Gefühle, die bis dahin aufgelöst wurden, bleiben aufgelöst. Die Patienten fühlen sich in der Regel auch hier bereits stabiler und erleichtert. Der Therapeut übernimmt bei einer MET-Traumatherapie konsequent die Führung und legt den jeweils nächsten Schritt der Behandlung fest. Er achtet in diesem Rahmen darauf, dass der Klient nicht zu schnell vorwärts geht, sondern nur eine schrittweise Annäherung vornimmt. So behält dieser die Kontrolle und verliert sich nicht in seinen Gefühlen.

Die MET-Traumatherapie ist grundsätzlich bei allen Traumata zu jeder Zeit anwendbar. Der Patient klopft während der Behandlung unentwegt die beiden Schlüsselbeinpunkte (Niere 27). Das ist bei einer MET-Behandlung generell der Fall. Die Behandlungsdauer erstreckt sich auf ein bis drei Sitzungen, die in der Regel eine Stunde dauern. Die emotionale Belastung des Klienten ist hierbei unter anderem durch die Leinwandtechnik und das Klopfen steuerbar. Es stellt sich meist rasch eine emotionale Stabilisierung und Kräftigung ein.

\section{Auflösung in Stufen: Annäherung, Bewusstwerdung, Vergebung}

Eine MET-Traumabehandlung besteht aus fünf Schritten:

1. Vorbereitungsphase: Da Traumapatienten meist Angst vor der Behandlung haben beziehungsweise vor dem, was dabei hochkommt, werden zunächst die Aufregung und die Ängste 
durch MET aufgelöst. Danach, wenn der Klient ruhig und entspannt ist, erlernt er die Leinwandtechnik.

2. Annäherung durch die Leinwandtechnik in Schwarz-Weiß (erste Sitzung): Das Bild wird in Schwarz-Weiß auf die Leinwand projiziert. Die Annäherung geschieht in 100-mSchritten, ab $100 \mathrm{~m}$ Entfernung in 10-m-Schritten und ab $10 \mathrm{~m}$ in $1-\mathrm{m}$-Schritten, bis das Bild sich in einem Meter Entfernung vor dem Klienten befindet.

3. Annäherung in Farbe: Das Bild wird in Farbe auf die imaginäre Leinwand projiziert. Manchmal geschieht dies auch schon in der ersten oder zweiten Sitzung. Weiteres Vorgehen siehe Schritt 2.

4. Schilderung des Traumas (zweite Sitzung): Der Klient erzählt Satz für Satz das traumatische Ereignis. Nach jedem Satz wird geprüft, ob irgendwelche emotionalen Belastungen hochkommen, die dann sofort aufgelöst werden.

5. Vergebung (dritte Sitzung): Hier geht es darum, mit der MET-Vergebungsarbeit mit sich selbst, aber auch mit dem Täter in Vergebung zu gehen.

Der Patient beklopft während der Sitzungen unentwegt leicht die beiden Schlüsselbeinpunkte (Punkt Nr. 6, siehe Abbildung 1), etwa ein Schlag pro Sekunde, immer so lange, bis das entsprechende Gefühl aufgelöst ist.

Im Gegensatz zu anderen Methoden beleuchtet die METKlopftherapie keine Problemursachen in der Vergangenheit. Es werden hingegen die Gefühle beklopft, die der Betroffene jetzt in Bezug auf sein Thema empfindet und als belastend erlebt. Hierdurch stellt sich häufig schnell ein Gefühl von Entspannung, Ruhe, Harmonie und innerem Frieden ein. MET bietet sich somit als schnell wirksame Kurzzeitbehandlung für die therapeutische Praxis an. Klienten können sie ergänzend als Selbsthilfemethode für alltägliche Probleme erlernen.

Die wichtigste Technik der MET-Klopftherapie ist das Beklopfen der sechs Energiepunkte:

1. am Beginn der Augenbraue

2. auf der Schläfe

3. unter dem Auge

4. unter der Nase

5. unterhalb der Unterlippe

6. unterhalb der Schlüsselbeingelenke

\section{THERAPIEBEISPIEL}

\section{Beispiel: Behandlung der Sprechangst}

Im Fall einer Sprechangst könnte der erste Klopfsatz lauten: „meine Angst, vor vielen Leuten zu sprechen”. Während des Klopfens löst sich dann diese Angst zunehmend auf. Dabei tritt möglicherweise eine weitere Angst in den Vordergrund, zum Beispiel „meine Angst, ausgelacht zu werden“. Vielleicht kommen noch weitere Gefühle hinzu, zum Beispiel Ärger, Trauer und Scham. Diese werden ebenfalls durch das Beklopfen der beiden Schlüsselbeinpunkte aufgelöst, sodass das Thema Sprechangst immer weiter in den Hintergrund tritt.
Man kann links oder rechts klopfen, auch beide Seiten gleichzeitig. Unter den Energiepunkten ist der Punkt Nr. 6 unter dem Schlüsselbein der wichtigste, weil sich hier immer alle negativen Gefühle auflösen. Er wird vom Klienten beidseits mit Daumen und Zeigefinger beklopft (etwa drei Schläge pro Sekunde), während er sich auf das belastende Gefühl konzentriert und den entsprechenden Klopfsatz ausspricht, zum Beispiel „meine Angst vor Krankheiten“, „meine Angst, gemobbt zu werden“ oder „meine Angst, bei der Prüfung (wieder) durchzufallen“. Er nennt also jeweils das bei Gedanken an eine bestimmte Situation auftauchende Gefühl.

\section{Weitere harmonisierende Methoden im Verbund}

Die Technik des Beklopfens lässt sich optional sehr gut mit weiteren Methoden kombinieren, die ebenfalls das Energieniveau erhöhen und die emotionale Lage harmonisieren.

\section{Atemgleichgewichtsübung: Gehirnhälften energetisch ausgleichen}

Die Atemgleichgewichtsübung gleicht die rechte und linke Gehirnhälfte energetisch aus und dauert nur etwa $2 \mathrm{~min}$. Sie bewirkt eine tiefe Entspannung und Zentrierung. Hierbei werden im Sit-

\section{AUS DER PATIENTENAKTE}

\section{Schulangst einer Jugendlichen nach Sitzung verschwunden}

Die 16-jährige Patientin erschien mit ihrer Mutter zum Termin. Sie litt unter Panikattacken in der Schule, hatte Angst, aus dem Haus zu gehen, sich von ihrer Mutter zu trennen, aber auch vor der nächsten Mathematikarbeit. Sie sah dem Ferienende und damit dem Schulbeginn mit großer Beklemmung entgegen. Zunächst beklopfte die Patientin ihre Angst, wieder eine Panikattacke zu erleiden. Nach nur einer Klopfrunde fing sie an zu lachen und bemerkte, das sei ja komisch, aber die Angst sei weg. Danach schob sich die nächste Angst in den Vordergrund, nämlich vor dem Ende der Ferien. Auch hier fing sie nach nur einer Klopfrunde wieder an zu lachen mit der Bemerkung, auch diese Angst sei jetzt verschwunden. Ich bat sie, noch einmal an ihre Schulsituation zu denken und nachzuspüren, ob es da jetzt noch irgendwelche Ängste gebe. Ja, da sei noch die Angst vor der nächsten Mathearbeit und dass sie nichts wüsste, obwohl sie alles gelernt habe. Beide Ängste wurden beklopft, mit demselben positiven Ergebnis. Nachdem alle mit der Schule verbundenen Ängste aufgelöst waren, erklärte sie auf Nachfrage: „Ich fühle mich trotzdem traurig, weil meine Mutter so viel Sorgen um mich hatte. "Auch diese Trauer wurde beklopft und aufgelöst, ebenso die anschließend auftauchenden Schuldgefühle gegenüber ihrem Bruder und ihrem Vater, weil sie die Mutter so beansprucht habe. Danach sprachen wir noch einmal sämtliche Angstauslöser durch. Bei keinem der Themen trat jedoch erneut Stress oder Angst auf. Die Patientin empfand darüber eine nachhaltige Erleichterung. Denn auch in der Folgezeit stellten sich keine Angstzustände mehr ein. 
zen die ausgestreckten Beine übereinander gekreuzt, links über rechts, ebenfalls die ausgestreckten Arme, jedoch rechts über links. Die Handflächen werden zueinander gedreht, die Finger verschränkt und die Arme derart gefaltet zum Brustbein herangezogen. Diese Position stammt aus dem Yoga und dient der Zentrierung und Entspannung. Gleichzeitig werden beide Gehirnhälften synchronisiert. Dann wird ruhig durch die Nase ein- und durch den Mund ausgeatmet. Beim Einatmen durch die Nase wird die Zunge leicht gegen den oberen Gaumen gedrückt. Beim Ausatmen durch den Mund entspannt der Klient die Zunge und sagt innerlich das Wort Gleichgewicht oder Balance.

\section{Thymusklopfen: Energiefluss und Lebensenergie stimulieren}

Die Thymusdrüse sitzt ca. $7 \mathrm{~cm}$ unterhalb der Halsgrube (Jugulum) und gilt als Zentrum unserer Lebensenergie (griech. thymos = Lebensenergie). Sie wird mit der flachen Hand oder leichten Faust sanft beklopft - auch etwa mit einem Schlag pro Sekunde -, wobei folgender Satz etwa fünfmal wiederholt wird: „Ich liebe und glaube, vertraue, bin dankbar und mutig." Durch das Beklopfen der Thymusdrüse wird der Energiefluss in den Meridianen harmonisiert, die Immunabwehr gestärkt und die Lebensenergie erhöht.

\section{Massieren des Heilenden Punktes: Selbstliebe stärken}

Der Heilende Punkt ist ein neurolymphatischer Reflexpunkt und befindet sich auf der linken Körperseite zwischen der 2. und 3. Rippe über dem Herz, ca. $10 \mathrm{~cm}$ links der Körpermitte. Er wird mit der flachen rechten Hand im Uhrzeigersinn massiert, wobei dreimal ein heilender Satz gesprochen wird wie: „Obwohl ich diese Angst habe, zu versagen, liebe und akzeptiere ich mich so, wie ich bin." Dies verbindet die Hinwendung und Anerkennung des Problems mit der Stärkung der Selbstliebe und -akzeptanz.

\section{Handrückenserie: Reflexpunkte und Augen koppeln}

Für die Handrückenserie wird der sogenannte Dreifache Erwärmer auf dem Handrücken (Meridianzuordnung Schilddrüse) leicht mit zwei bis drei Fingern der anderen Hand ca. 10- bis 15mal beklopft, wieder mit etwa einem Schlag pro Sekunde, während man nach unten rechts und unten links blickt, die Augen nach links und rechts kreisen lässt, eine Tonfolge summt oder schnell zählt (Synchronisation linke und rechte Gehirnhälfte). Diese Übung bewirkt eine Harmonisierung des Energieflusses in den Meridianen.

Dieser Artikel ist online zu finden: http://dx.doi.org/10.1055/a-1082-9604

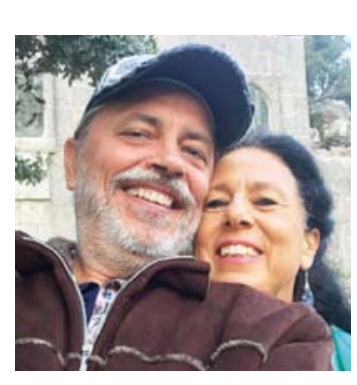

\section{RAINER UND REGINA FRANKE}

Rainer-Michael Franke (geb. 1953) ist Diplom-Psychologe, Gestaltpsychotherapeut und seit 30 Jahren im Bereich Psychotherapie, Coaching, Seminare, Supervision und Ausbildung tätig. Seit 2001 hat er die MET-Klopftherapie entwickelt. Er ist Autor von acht Büchern zur MET-Klopftherapie, unter anderem: Franke RM, Franke R: MET-Klopftherapie in der Praxis. Stuttgart: Haug; 2019.

Regina Franke (geb. 1955) ist Heilpraktikerin und hat seit 2001 zusammen mit Rainer-Michael Franke die MET-Klopftherapie entwickelt. Sie ist Autorin von sieben MET-Büchern und führt zusammen mit ihrem Mann die Franke Akademie in Sa Ràpita, Mallorca.

E-Mail: info@franke-akademie.de

Internet: www.franke-akademie.de 